EPJ Web of Conferences 41, 09014 (2013)

DOI: 10.1051/epjconf/20134109014

(C) Owned by the authors, published by EDP Sciences, 2013

\title{
Ultrafast infrared near-field molecular nano-spectroscopy
}

\author{
Xiaoji Xu, Ian M. Craig, Matthias Rang, and Markus B. Raschke \\ Department of Physics, Department of Chemistry, and JILA, University of Colorado, \\ Boulder, CO 80303, USA
}

\begin{abstract}
We demonstrate molecular radiative infrared vibrational free-induction decay on the nano-scale and its control via near-field coupling between the transient molecular polarization and optical antenna properties of the metallic scanning near-field probe tip. This allows for pushing the sensitivity of infrared vibrational spectroscopy into the single molecule regime.
\end{abstract}

\section{Introduction}

The extension of ultrafast spectroscopy to the nanoscale to access the underlying spatial inhomogeneities of far-field ensemble averaging spectroscopy has been a long-standing challenge $[1,2]$. Its application to infrared vibrational molecular spectroscopy is particularly underdeveloped yet highly desirable for its chemical sensitivity and access to chemical dynamics. In this work we combine scattering scanning near-field optical microscopy ( $s$-SNOM) with femtosecond infrared spectroscopy for ultrafast molecular vibrational nano-spectroscopy in the mid-infrared. The metallic scanning probe tip provides a nanoscale field localization and near-field coupling via evanescent modes with the induced coherent vibrational polarization. Via its enhanced coupling to the far-field electromagnetic density of states this gives rise to tip scattered radiative emission of the vibrational molecular free-induction decay (FID) in competition against the rapid intra- and inter-molecular nonradiative decay in the condensed phase. With increasing degree of near-field coupling between molecular excitation and metallic antenna tip we can control the rate of mode transfer and thus controllably enhance the vibrational relaxation from $\mathrm{T}_{2}{ }^{\mathrm{FF}}=720$ fs to $\mathrm{T}_{2}{ }^{\mathrm{NF}}=250$ fs. We demonstrate a drastic tip-enhanced increase in sensitivity of ultrafast IR spectroscopy by $>10^{4}$ probing ensembles as low as 100 vibrational oscillators reaching the realm of single molecule IR spectroscopy. This approach allows for spatio-temporal imaging on nanometer length and femtosecond time scales including extension to multi-dimensions IR nano-spectroscopy [2].

\section{Method}

The schematic of the experiment is shown in Fig. 1a). Mid-IR radiation tunable from 5-10 $\mu \mathrm{m}$ with pulse duration of $220 \mathrm{fs}$ is generated by difference frequency generation from a high power Ti:S oscillator pumped OPO. The IR beam is focused onto the apex of a metallic scanning probe tip of a modified atomic force microscope operating in dynamic force mode. The tip-scattered IR light is collected in epi-detection, interferometrically heterodyned with the reference field of variable time

This is an Open Access article distributed under the terms of the Creative Commons Attribution License 2.0, which permits unrestricted use, distribution, and reproduction in any medium, provided the original work is properly cited. 
delay, detected by an MCT detector, and demodulated using a lock-in detector at higher harmonics of the tip dither frequency for near-field signal discrimination. Multiple harmonics are recorded simultaneously while scanning the reference mirror for heterodyne recoding of the interferograms. As samples polytetrafluoroethylene (PTFE) serve as a model system for its high transition dipole moment of the $\mathrm{C}-\mathrm{F}$ bond and high oscillator density. For spatially resolved studies self-assembled monolayers of mercaptohexadecanoic acid (MHDA) on gold are used.
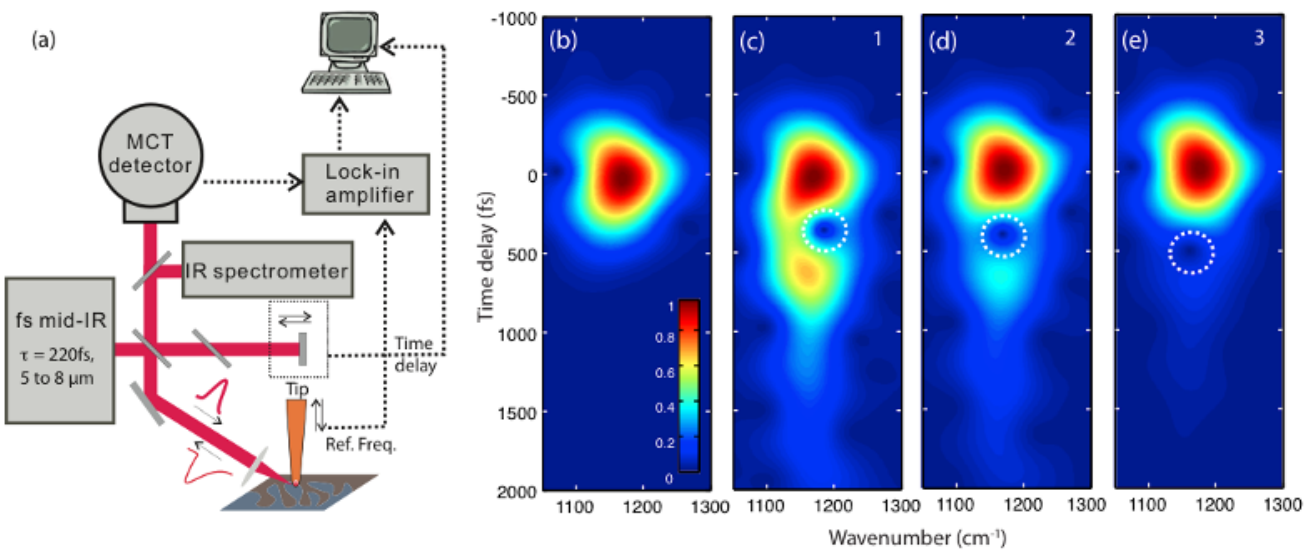

Fig. 1. Near-field control of vibrational coherence: a) Experimental setup for infrared vibrational scattering scanning near-field otical microscopy (s-SNOM). Spectrogram of $220 \mathrm{fs}$ mid-IR laser pulse (b), and radiative tip-scattered near-field free induction decay of molecular polarization in $\mathrm{C}-\mathrm{F}$. With increasing tip-sample coupling and polarization transfer to the tip this leads to an associated decrease of the FID decay from 570 to $250 \mathrm{fs}$ (c-d) [6].

\section{Results}

Fig. 1a) shows the detected tip-scattered interferogram at third cantilever harmonics with Au as nonresonant reference sample after wavelet analysis with a $200 \mathrm{fs}$ gate function, representing the driving laser field in the near-field of the apex with laser tuned to $1170 \mathrm{~cm}^{-1}$ center frequency $\left(70 \mathrm{~cm}^{-1}\right.$ bandwidth). Fig. 1c) shows the corresponding results for the PTFE sample. Radiative resonant freeinduction decay dominated by the symmetric C-F stretch mode $\left(v_{1}=1160 \mathrm{~cm}^{-1}\right)$ is discerned with a dephasing time of $\mathrm{T}_{2}=(570 \pm 30) \mathrm{fs}$ and enhanced compared to non-radiatively dominated far-field response of $\mathrm{T}_{2}=720 \mathrm{fs}$. With increasing tip-sample coupling (c-e), and increase in efficiency of mode transfer from molecular polarization to the tip, further decrease in vibrational lifetime via $T_{2}=$ $(480 \pm 40)$ fs $(d)$ to $T_{2}=(250 \pm 60)$ fs $(e)$ is achieved. The results can be described using a dipoledipole model reproducing FID behaviour and interference features (dashed white circle) and their evolution with increasing degree of coupling [6].

The resulting increase in sensitivity and spatial localization of the FID signal allows for nanometer spatially resolved imaging with unprecedented sensitivity. Fig. 2 (a-d) demonstrate the molecular monolayer sensitivity of the MHDA SAMs on gold, probing the $\mathrm{C}=\mathrm{O}$ resonance $[67$. With a spatial resolution of $30 \mathrm{~nm}$ and a molecular density of $1 / \mathrm{nm}^{2}$ with the contrast and $\mathrm{s} / \mathrm{n}$ obtained this demonstrates a sensitivity of 100 vibrational oscillators or less, exceeding far-field IR spectroscopy sensitivity by $10^{4}$.

Our experiments are the conceptual extension of surface-enhanced infrared absorption (SEIRA) [3], phonon IR s-SNOM [4] and tip-enhanced fluorescence [5] into the ultrafast regime. The observed decrease in $\mathrm{C}-\mathrm{F}$ decoherence time from far-field to tip-enhanced near-field is summarized in Fig. 2e) together with a schematics illustrating the underlying decay channels. Without near-field coupling the vibrational relaxation proceeds primarily through non-radiative intra- and intermolecular coupling corresponding to $\mathrm{T}_{2}{ }^{\mathrm{FF}}=1 / \Gamma_{\text {nrad }}$. With increasing coupling of the metallic tip 
to the excited molecule coherent polarization transfer into the tip results in a decrease in molecular coherence time. This energy is dissipated via non-radiative scattering in the metal (ohmic loss) and detectable radiative emission $\Gamma_{\text {tip }}=\Gamma_{\text {ohmic }}+\Gamma_{\text {rad }}$.
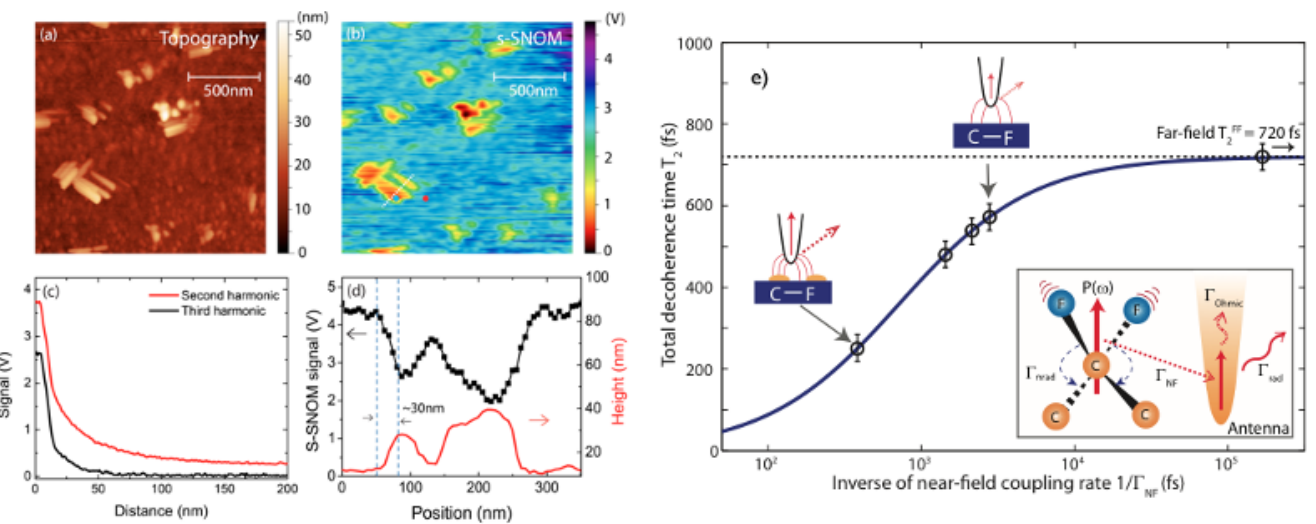

Fig. 2. Ultrahigh spatial resolution tip-coupled FID imaging and sensitivity: Topography (a) and integrated FID image (b) of $\mathrm{C}=\mathrm{O}$ mode of MHDA monolayer, with few 10's nm near-field FID localization (c) and resolution (d) [6]. Model of microscopic optical tip-molecule interaction (e): Decrease of measured nearfield vibrational $\mathrm{C}-\mathrm{F}$ dephasing $\mathrm{T}_{2}$ with increasing degree of tip-sample coupling plotted as $1 / \mathrm{T}_{2}$ (total) $=$ $1 / \mathrm{T}_{2}{ }^{\mathrm{FF}}$ (non-rad) $+\Gamma_{\mathrm{NF}}$. The dipole-dipole coupled mode transfer into the tip/antenna subsequently decays non-radiatively (ohmic loss) or by far-field radiation (detected signal) [7].

In summary we have demonstrated the extension of ultrafast coherent infrared spectroscopy into the near-field regime. We take advantage of the optical antenna properties and field localization of the metallic scanning probe tip. They mediate the mode mismatch between the near-field molecular coherent polarization and far-field density of states succeeding in the competition against the otherwise dominant non-radiative decoherence. This results in enhanced sensitivity of coherent IR spectroscopy and its nano-scale implementation. We expect that the generalization of this approach to any form of linear and nonlinear wavemixing techniques including multi-dimensional spectroscopy will allow for vibrational quantum coherent control and full spatio-temporal imaging of vibrational dynamics on the nanoscale.

[1] A. Kubo, N. Pontius, and H. Petek, Nano Letters 7, 470 (2007).

[2] M. Aeschlimann, M. Bauer, D. Bayer, T. Brixner, F. J. García de Abajo, W. Pfeiffer, M. Rohmer, C. Spindler, and F. Steeb, Nature, 446, 301 (2007).

[3] F.Neubrech,A.Pucci,T.W.Cornelius,S.Karim,A.Garca-Etxarri,andJ.Aizpurua, Phys. Rev. Lett. 101, 157403 (2008).

[4] S. Amarie, T. Ganz, and F. Keilmann, Optics Express 17, 21794 (2009).

[5] P. Anger, P. Bharadwaj, and L. Novotny, Phys. Rev. Lett. 96, 113002 (2006).

[6] X. G. Xu and M. B. Raschke (under review).

[7] X. G. Xu, M. Rang, I. M. Craig, and M. B. Raschke, J. Phys. Chem. Lett. 3, 1836 (2012). 